\title{
Finite-Time Stability of Neutral Fractional Time-Delay Systems via Generalized Gronwalls Inequality
}

\author{
Pang Denghao and Jiang Wei \\ School of Mathematical Sciences, Anhui University, Hefei 230039, China \\ Correspondence should be addressed to Jiang Wei; jiangwei89018@126.com
}

Received 22 October 2013; Accepted 2 January 2014; Published 23 February 2014

Academic Editor: Irena Rachůnková

Copyright ( 2014 P. Denghao and J. Wei. This is an open access article distributed under the Creative Commons Attribution License, which permits unrestricted use, distribution, and reproduction in any medium, provided the original work is properly cited.

This paper studies the finite-time stability of neutral fractional time-delay systems. With the generalized Gronwall inequality, sufficient conditions of the finite-time stability are obtained for the particular class of neutral fractional time-delay systems.

\section{Introduction}

In this paper, we consider a neutral fractional time-delay system:

$$
\begin{gathered}
{ }^{c} \mathfrak{D}^{\alpha} x(t)=A x(t)+B x(t-\tau)+C{ }^{c} \mathfrak{D}^{\alpha} x(t-\tau), \\
t \in[0, T], \\
x(t)=\varphi(t), \quad t \in[-\tau, 0],
\end{gathered}
$$

where ${ }^{c} \mathfrak{D}^{\alpha}$ denotes the Caputo fractional derivative of order $0<\alpha \leq 1$, the vector function $x(t) \in R^{n}, A, B, C$ are constant system matrices of appropriate dimensions, the constant parameter $\tau>0$ represents the delay argument, and $\varphi(t)$ is a given continuously differentiable function on $[-\tau, 0]$.

The neutral time-delay systems have received increasing attention (see [1-5]) due to their successful applications in population ecology, distributed networks containing lossless transmission lines, heat exchangers, robots in contact with rigid environments, partial element equivalent circuit (PEEC), the control of constrained manipulators with timedelay measurements, the systems which need the information of the past state variables, and so on.

Recently, with the development of theories of fractional differential equations (see [6-9]), there has been a surge in the study of neutral fractional time-delay systems (see [10-12]). In particular, the problem of stability analysis of such systems has been one of the most interesting topics in control theory because stability analysis is one of the most important issues for control systems (see [13-16]). But stability of these systems proves to be a more complex issue because the systems involve the derivative of the time-delayed state and the existence of time-delay is frequently the source of instability although this problem has been investigated for time-delay systems over many years. In the previous literatures, many scholars have utilized the Lyapunov technique, characteristic equation method, state solution approach, or Gronwall's approach to derive sufficient conditions for stability of the systems. In this paper, motivated by the papers $[17,18]$, we discuss the stability of the neutral fractional system with delay via generalized Gronwall's approach.

The organization of this paper is as follows. In Section 2, we summarize some notations and give preliminary results which will be used in this paper. In Section 3, we present our main results.

\section{Preliminaries and Lemmas}

Let us start with some definitions and lemmas which are used throughout this paper.

Definition 1 (see [7]). Euler's gamma function is defined as

$$
\Gamma(z)=\int_{0}^{\infty} e^{-t} t^{z-1} d t, \quad z \in \mathbb{C},
$$

where $\mathbb{C}$ denotes the complex plane. 
Remark 2 (see [7]). (i) $\Gamma(z+1)=z \Gamma(z), z \in \mathbb{C}$; and for $n \in$ $\mathbb{Z}^{+}, \Gamma(n+1)=n(n-1) !=n !$

$$
2^{2 n} n !
$$$$
\begin{aligned}
& \text { (ii) } \Gamma(z) \Gamma(z+(1 / 2))=\sqrt{\pi} 2^{1-2 z} \Gamma(2 z), 2 z \neq 0,-1,-2, \ldots ; \\
& \text { (ii) } \Gamma(n+(1 / 2))=\sqrt{\pi} \Gamma(2 n+1) / 2^{2 n} \Gamma(n+1)=\sqrt{\pi}(2 n) ! /
\end{aligned}
$$

Definition 3 (see [7]). The fractional integral of order $\alpha$ with the lower limit zero for any function $f(t) \in C([0,+\infty), R)$, $t \geq 0$, is defined as

$$
\begin{aligned}
I^{\alpha} f(t) & =\lim _{\substack{h \rightarrow 0 \\
n h=t}} h^{\alpha} \sum_{r=0}^{n}\left[\begin{array}{l}
\alpha \\
r
\end{array}\right] f(t-r h) \\
& =\frac{1}{\Gamma(\alpha)} \int_{0}^{t}(t-\theta)^{\alpha-1} f(\theta) d \theta, \quad \alpha>0,
\end{aligned}
$$

where $\left[\begin{array}{c}\alpha \\ r\end{array}\right]=\alpha(\alpha+1) \cdots(\alpha+r-1) / r$ !, and $\Gamma(\cdot)$ is the gamma function.

Definition 4 (see [7]). The Riemann-Liouville derivative of order $\alpha$ with the lower limit zero for any function $f(t) \in$ $C([0,+\infty), R), t \geq 0$, is defined as

$$
\begin{array}{r}
{ }^{l} \mathfrak{D}^{\alpha} f(t)=\frac{1}{\Gamma(n-\alpha)} \frac{d^{n}}{d t^{n}} \int_{0}^{t}(t-\theta)^{n-\alpha-1} f(\theta) d \theta, \\
n-1<\alpha<n .
\end{array}
$$

Definition 5 (see [7]). The Caputo derivative of order $\alpha$ for any function $f(t) \in C^{n}([0,+\infty), R), t \geq 0$, is defined as

$$
\begin{aligned}
{ }^{c} \mathfrak{D}^{\alpha} f(t) & =\frac{1}{\Gamma(n-\alpha)} \int_{0}^{t}(t-\theta)^{n-\alpha-1} f^{(n)}(\theta) d \theta \\
& =I^{n-\alpha} f(t), \quad n-1<\alpha<n .
\end{aligned}
$$

Remark 6 (see [7]). (i) If a function $f(t) \in C^{n}([0,+\infty), R)$, $t \geq 0$, then ${ }^{c} \mathfrak{D}^{\alpha} f(t)={ }^{l} \mathfrak{D}^{\alpha} f(t)-\sum_{k=0}^{n-1}\left(f^{(k)}(0) / \Gamma(k-\alpha+\right.$ 1)) $t^{k-\alpha}$;

(ii) ${ }^{c} \mathfrak{D}^{\alpha} f(t)=0, f(t)=C$, and $C$ is a constant.

Definition 7 (see [9]). The Mittag-Leffler function in two parameters is defined as

$$
E_{\alpha, \beta}(z)=\sum_{k=0}^{\infty} \frac{(z)^{k}}{\Gamma(\alpha k+\beta)}, \quad z \in \mathbb{C}
$$

where $\alpha>0, \beta>0$ and $z \in \mathbb{C}$.

Remark 8 (see [9]). (i) For $\beta=1, E_{\alpha, 1}\left(\lambda z^{\alpha}\right)=E_{\alpha}\left(\lambda z^{\alpha}\right)=$ $\sum_{k=0}^{\infty}\left(\lambda^{k}\left(z^{\alpha}\right)^{k} / \Gamma(\alpha k+1)\right)$, and $E_{(1,1)}(z)=e^{z}, z \in \mathbb{C}$;

(ii) For $\beta=1$, the matrix extension of the aforementioned Mittag-Leffler function has the following representation: $E_{\alpha}\left(A t^{\alpha}\right)=\sum_{k=0}^{\infty}\left(\left(A^{k}\left(t^{\alpha}\right)^{k} / \Gamma(\alpha k+1)\right), z \in \mathbb{C}\right.$, and ${ }^{c} \mathfrak{D}^{\alpha} E_{\alpha}\left(A t^{\alpha}\right)=A E_{\alpha}\left(A t^{\alpha}\right)$.

Lemma 9 (see [19] generalized Gronwall's inequality). Suppose $x(t), a(t)$ are nonnegative and local integrable on $0 \leq t<$ $T$, some $T \leq \infty$, and $g(t)$ is a nonnegative, nondecreasing continuous function defined on $0 \leq t<T ; g(t) \leq M=$ constant $\alpha>0$ with

$$
x(t) \leq a(t)+g(t) \int_{0}^{t}(t-s)^{\alpha-1} x(s) d s
$$

on this interval. Then

$$
x(t) \leq a(t)+g(t) \int_{0}^{t}\left[\sum_{n=1}^{\infty} \frac{(g(t) \Gamma(\alpha))^{n}}{\Gamma(n \alpha)}(t-s)^{n \alpha-1} a(s)\right] d s,
$$

$0 \leq t<T$

Lemma 10 (see [19]). Under the hypothesis of Theorem 13, let $a(t)$ be a nondecreasing function on $[0, T)$. Then

$$
x(t) \leq a(t) E_{\alpha}\left(g(t) \Gamma(\alpha) t^{\alpha}\right),
$$

where $E_{\alpha}$ is the Mittag-Leffler function.

\section{Main Results}

In this section, we discuss some problems of the neutral fractional time-delay system (1).

Let us denote by $C([a, b])$ the space of all continuous real functions defined on $[a, b]$ and by $C\left([a, b], R^{n}\right)$ the Banach space of continuous functions mapping the interval $[a, b]$ into $R^{n}$ with the topology of uniform convergence. Let $C=$ $C\left([-\tau, 0], R^{n}\right),[a, b]=[-\tau, 0]$, and designate the norm of an element $\varphi$ in $C$ by

$$
\|\varphi\|=\sup _{-\tau \leq t \leq 0}\|\varphi(t)\|
$$

Let $X=C\left([-\tau, T], R^{n}\right)$ and $x(t)=\varphi(t), t \in[-\tau, 0]$ be equipped with the norm

$$
\begin{array}{r}
\|x(t)\|:=\sup _{0 \leq t \leq T} x(t), \\
\left\|x_{t}\right\|:=\|x(t+\theta)\|:=\sup _{-\tau \leq \theta \leq 0}\|x(t+\theta)\|,
\end{array}
$$

$\forall x \in X$

where obviously $\|x(t)\| \leq\left\|x_{t}\right\|$.

Let $v_{\max }(\cdot)$ be the largest singular value of matrix $(\cdot)$, namely,

$$
v_{\max }^{A B}=v_{\max }(A)+v_{\max }(B) .
$$

For convenience, we denote $\nu_{\max }(A)$ by $\nu_{0}, v_{\max }(B)$ by $\nu_{1}$, $v_{\max }(C)$ by $v_{2}, v_{\max }(D)$ by $v_{3}$, and $v_{\max }(A B)$ by $v_{01}$, respectively.

Definition 11 (see [18]). The system given by (1) and satisfying initial condition $x(t)=\varphi(t)$, for $t \in[-\tau, 0]$, is finite stable with respect to $\left\{t_{0}, \delta, \epsilon, J\right\}, \delta<\epsilon, J=\left[t_{0}, t_{0}+T\right]$ if and only if

$$
\|\varphi\|<\delta
$$

implies

$$
\|x(t)\|<\epsilon, \quad \forall t \in J .
$$


Theorem 12. If $x(t)=x(t, \varphi)$ is a solution of the systems (1), then there exists a positive constant $v$ such that

$$
\|x(t)\| \leq\left(1+2 v_{2}\right)\|\varphi\| E_{\alpha}\left(\nu t^{\alpha}\right) \quad \forall t \in J=[0, T] .
$$

Proof. According to the properties of the fractional order $0<$ $\alpha<1$, one can obtain a solution in the form of the equivalent Volterra integral equation [12]:

$$
\begin{aligned}
x(t)= & x(0)+C(x(t-\tau)-x(-\tau)) \\
& +\frac{1}{\Gamma(\alpha)} \int_{0}^{t}(t-s)^{\alpha-1}(A x(s)+B x(s-\tau)) d s \\
= & \varphi(0)+C \varphi(-\tau)+C x(t-\tau) \\
& +\frac{1}{\Gamma(\alpha)} \int_{0}^{t}(t-s)^{\alpha-1}(A x(s)+B x(s-\tau)) d s, \quad t \geq 0 .
\end{aligned}
$$

Using appropriate property of the norm $\|(\cdot)\|$ in (16) and applying (10), it follows that

$$
\begin{aligned}
\|x(t)\| \leq & \|\varphi(0)\|+\|C \varphi(-\tau)\|+\|C x(t-\tau)\| \\
& +\frac{1}{\Gamma(\alpha)} \int_{0}^{t}|t-s|^{\alpha-1}\|A x(s)+B x(s-\tau)\| d s \\
\leq & (1+\|C\|)\|\varphi\|+\|C x(t-\tau)\| \\
& +\frac{1}{\Gamma(\alpha)} \int_{0}^{t}|t-s|^{\alpha-1}\|A x(s)+B x(s-\tau)\| d s \\
\leq & (1+\|C\|)\|\varphi\|+\|C\|\|x(t-\tau)\| \\
& +\frac{1}{\Gamma(\alpha)} \int_{0}^{t}|t-s|^{\alpha-1}\|A\|\|x(s)\| d s \\
& +\frac{1}{\Gamma(\alpha)} \int_{0}^{t}|t-s|^{\alpha-1}\|B\|\|x(s-\tau)\| d s \\
\leq & \left(1+v_{2}\right)\|\varphi\|+v_{2}\|x(t-\tau)\| \\
& +\frac{v_{0}}{\Gamma(\alpha)} \int_{0}^{t}|t-s|^{\alpha-1}\|x(s)\| d s \\
& +\frac{v_{1}}{\Gamma(\alpha)} \int_{0}^{t}|t-s|^{\alpha-1}\|x(s-\tau)\| d s, \quad t \geq 0 .
\end{aligned}
$$

For $0 \leq t \leq \tau,\|x(t-\tau)\| \leq\|\varphi\|$, (17) can be rewritten as

$$
\begin{aligned}
\|x(t)\| \leq & \left(1+2 v_{2}\right)\|\varphi\|+\frac{v_{0}}{\Gamma(\alpha)} \int_{0}^{t}|t-s|^{\alpha-1}\|x(s)\| d s \\
& +\frac{v_{1}}{\Gamma(\alpha)} \int_{0}^{t}|t-s|^{\alpha-1}\|x(s-\tau)\| d s, \quad 0 \leq t \leq \tau .
\end{aligned}
$$

From Definition 3, we can see $I^{\alpha} f(t)$ is an increasing function of $t$ if $f(t)>0$. So $\left(v_{0} / \Gamma(\alpha)\right) \int_{0}^{t}|t-s|^{\alpha-1}\|x(s)\| d s$ and $\left(\nu_{1} / \Gamma(\alpha)\right) \int_{0}^{t}|t-s|^{\alpha-1}\|x(s-\tau)\| d s$ are both increasing functions with regard to $t$. Taking into account (18) and (11), it yields that

$$
\begin{aligned}
\left\|x_{t}\right\| \leq & \left(1+2 v_{2}\right)\|\varphi\|+\frac{v_{0}}{\Gamma(\alpha)} \int_{0}^{t}|t-s|^{\alpha-1}\left\|x_{s}\right\| d s \\
& +\frac{v_{1}}{\Gamma(\alpha)} \int_{0}^{t}|t-s|^{\alpha-1}\left\|x_{s}\right\| d s \\
\leq & \left(1+2 v_{2}\right)\|\varphi\|+\frac{v_{01}}{\Gamma(\alpha)} \int_{0}^{t}|t-s|^{\alpha-1}\left\|x_{s}\right\| d s, \\
& 0 \leq t \leq \tau .
\end{aligned}
$$

Let us introduce a function $a(t)$ such as

$$
a(t)=\left(1+2 v_{2}\right)\|\varphi\|,
$$

where the function $a(t)$ is nondecreasing apparently.

Now, with the corollary of the generalized Gronwall inequality (9), we can obtain

$$
\left\|x_{t}\right\| \leq\left(1+2 v_{2}\right)\|\varphi\| E_{\alpha}\left(v_{01} t^{\alpha}\right), \quad 0 \leq t \leq \tau .
$$
mate:

Similarly, the same argument implies the following esti-

$$
\begin{array}{r}
\left\|x_{t}\right\| \leq\left(1+2 v_{2}\right)\left\|x_{\tau_{0}}\right\| E_{\alpha}\left(v_{01}\left(t-\tau_{0}\right)^{\alpha}\right), \\
\tau_{0} \leq t \leq \tau_{0}+\tau, \quad \tau_{0} \geq 0 .
\end{array}
$$

From Definition 7, we know that the Mittag-Leffler function $E_{\alpha}(t)$ is an increasing function with regard to $t$. Therefore, there exists $v>v_{01}$ such that $E_{\alpha}\left(\nu \tau^{\alpha}\right)>E_{\alpha}\left(v_{01} \tau^{\alpha}\right)$ and $E_{\alpha}\left(\nu(t-\tau)^{\alpha}\right) E_{\alpha}\left(\nu_{01} \tau^{\alpha}\right) / E_{\alpha}\left(\nu t^{\alpha}\right)<1 /\left(1+2 v_{2}\right)$.

Relationships (21) and (22) suggest the following general expression:

$$
\left\|x_{t}\right\| \leq\left(1+2 v_{2}\right)\|\varphi\| E_{\alpha}\left(\nu t^{\alpha}\right), \quad 0 \leq t \leq n \tau \leq T .
$$

To prove formula (23) by induction we have to show that it holds for $n=1$ because of formula (21) and if it holds for $n=$ $k$, then it holds also for $n=k+1$. Indeed, for $t \in[\tau,(k+1) \tau]$, $t-\tau \in[0, k \tau]$; on the one hand using formula (22),we have

$$
\left\|x_{t}\right\| \leq\left(1+2 v_{2}\right)\left\|x_{t-\tau}\right\| E_{\alpha}\left(v_{01} \tau^{\alpha}\right) .
$$

On the other hand, using formula (23) we obtain

$$
\left\|x_{t-\tau}\right\| \leq\left(1+2 v_{2}\right)\|\varphi\| E_{\alpha}\left(\nu(t-\tau)^{\alpha}\right) .
$$

Taking into account (24) and (25) we conclude that

$$
\begin{aligned}
\left\|x_{t}\right\| \leq & \left(1+2 v_{2}\right)\left[\left(1+2 v_{2}\right)\|\varphi\| E_{\alpha}\left(\nu(t-\tau)^{\alpha}\right)\right] E_{\alpha}\left(v_{01} \tau^{\alpha}\right) \\
= & \left(1+2 v_{2}\right)\|\varphi\| E_{\alpha}\left(\nu t^{\alpha}\right) \\
& \times \frac{\left(1+2 v_{2}\right) E_{\alpha}\left(\nu(t-\tau)^{\alpha}\right) E_{\alpha}\left(v_{01} \tau^{\alpha}\right)}{E_{\alpha}\left(\nu t^{\alpha}\right)} \\
\leq & \left(1+2 v_{2}\right)\|\varphi\| E_{\alpha}\left(\nu t^{\alpha}\right) .
\end{aligned}
$$


That is,

$$
\|x(t)\| \leq\left\|x_{t}\right\| \leq\left(1+2 v_{2}\right)\|\varphi\| E_{\alpha}\left(v t^{\alpha}\right) .
$$

The proof is completed.

Theorem 13. The neutral fractional time-delay systems given by (1) are finite-time stable with respect to $\{0, \delta, \epsilon, J\}, \delta<\epsilon$, if the following condition is satisfied:

$$
\left(1+2 v_{2}\right) E_{\alpha}\left(v t^{\alpha}\right) \leq \frac{\epsilon}{\delta}, \quad \forall t \in J=[0, T] .
$$

Proof. From Theorem 12 we obtain

$$
\|x(t)\| \leq\left(1+2 v_{2}\right)\|\varphi\| E_{\alpha}\left(v t^{\alpha}\right) .
$$

Hence, using Definition 11 and the basic condition of Theorem 13, it follows that

$$
\|x(t)\|<\epsilon, \quad \forall t \in J=[0, T] .
$$

The proof is completed.

\section{Conflict of Interests}

The authors declare that there is no conflict of interests regarding the publication of this paper.

\section{Acknowledgments}

The authors sincerely thank the reviewers for their valuable suggestions and useful comments that have led to the present improved version of the original manuscript. This research was jointly supported by National Natural Science Foundation of China (no. 11371027 and no. 11071001), Doctoral Fund of Ministry of Education of China (no. 20093401110001) and Major Program of Educational Commission of Anhui Province of China (no. KJ2011A020).

\section{References}

[1] K. Zhang and D. Q. Cao, "Further results on asymptotic stability of linear neutral systems with multiple delays," Journal of the Franklin Institute. Engineering and Applied Mathematics, vol. 344, no. 6, pp. 858-866, 2007.

[2] D. Q. Cao and P. He, "Stability criteria of linear neutral systems with a single delay," Applied Mathematics and Computation, vol. 148, no. 1, pp. 135-143, 2004.

[3] P. T. Nam and V. N. Phat, "An improved stability criterion for a class of neutral differential equations," Applied Mathematics Letters, vol. 22, no. 1, pp. 31-35, 2009.

[4] P. Balasubramaniam, R. Krishnasamy, and R. Rakkiyappan, "Delay-dependent stability of neutral systems with timevarying delays using delay-decomposition approach," Applied Mathematical Modelling, vol. 36, no. 5, pp. 2253-2261, 2012.

[5] F. Wang, "Exponential asymptotic stability for nonlinear neutral systems with multiple delays," Nonlinear Analysis: Real World Applications, vol. 8, no. 1, pp. 312-322, 2007.

[6] K. S. Miller and B. Ross, An Introduction to the Fractional Calculus and Fractional Differential Equations, John Wiley \& Sons, New York, NY, USA, 1993.
[7] I. Podlubny, Fractional Differential Equations, Academic Press, San Diego, Calif, USA, 1999.

[8] A. Anatoly Kilbas and H. M. Srivastava, Theory and Applications of Fractional Differential Equations, Elsevier, Amsterdam, The Netherlands, 2006.

[9] T. Kaczorek, Selected Problems of Fractional Systems Theory, Springer, Berlin, Germany, 2011.

[10] Y. Zhou, F. Jiao, and J. Li, "Existence and uniqueness for fractional neutral differential equations with infinite delay," Nonlinear Analysis: Theory, Methods \& Applications, vol. 71, no. 7-8, pp. 3249-3256, 2009.

[11] S. Liu, X. Li, W. Jiang, and X. Zhou, "Mittag-Leffler stability of nonlinear fractional neutral singular systems," Communications in Nonlinear Science and Numerical Simulation, vol. 17, no. 10, pp. 3961-3966, 2012.

[12] X.-F. Zhou, J. Wei, and L.-G. Hu, "Controllability of a fractional linear time-invariant neutral dynamical system," Applied Mathematics Letters, vol. 26, no. 4, pp. 418-424, 2013.

[13] S. Liu and W. Jiang, "Asymptotic stability of nonlinear descriptor systems with infinite delays," Annals of Differential Equations, vol. 26, no. 2, pp. 174-180, 2010.

[14] Z. Zhang and J. Wei, "Some results of the degenerate fractional differential system with delay," Computers \& Mathematics with Applications, vol. 62, no. 3, pp. 1284-1291, 2011.

[15] M. P. Lazarević, "Finite time stability analysis of $P D^{\alpha}$ fractional control of robotic time-delay systems," Mechanics Research Communications, vol. 33, no. 2, pp. 269-279, 2006.

[16] C. Bonnet and J. R. Partington, "Analysis of fractional delay systems of retarded and neutral type," Automatica, vol. 38, no. 8, pp. 1133-1138, 2002.

[17] X. Zhang, "Some results of linear fractional order time-delay system," Applied Mathematics and Computation, vol. 197, no. 1, pp. 407-411, 2008.

[18] M. P. Lazarević and A. M. Spasić, "Finite-time stability analysis of fractional order time-delay systems: Gronwall's approach," Mathematical and Computer Modelling, vol. 49, no. 3-4, pp. 475481, 2009.

[19] H. Ye, J. Gao, and Y. Ding, "A generalized Gronwall inequality and its application to a fractional differential equation," Journal of Mathematical Analysis and Applications, vol. 328, no. 2, pp. 1075-1081, 2007. 


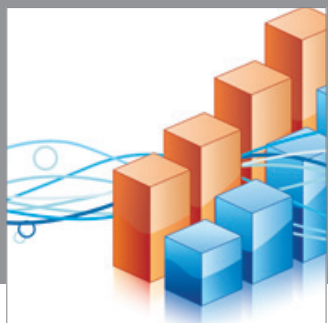

Advances in

Operations Research

mansans

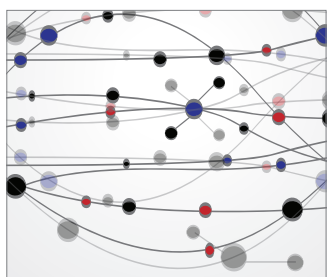

The Scientific World Journal
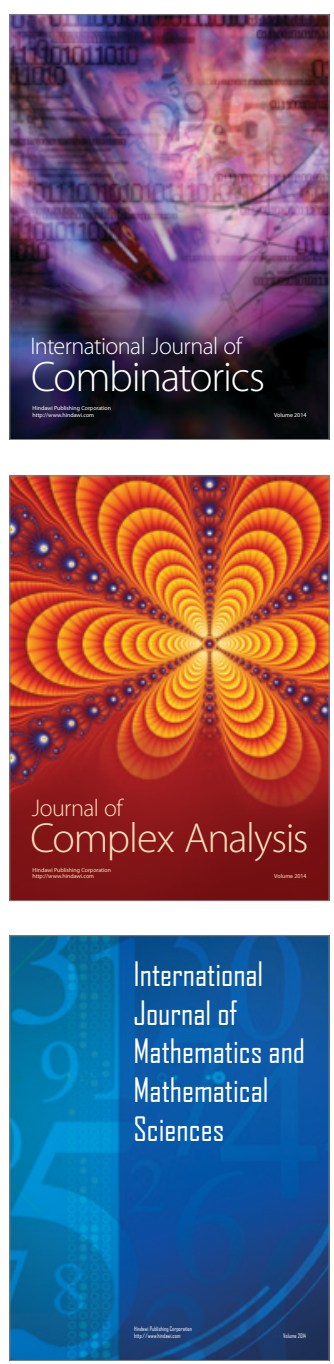
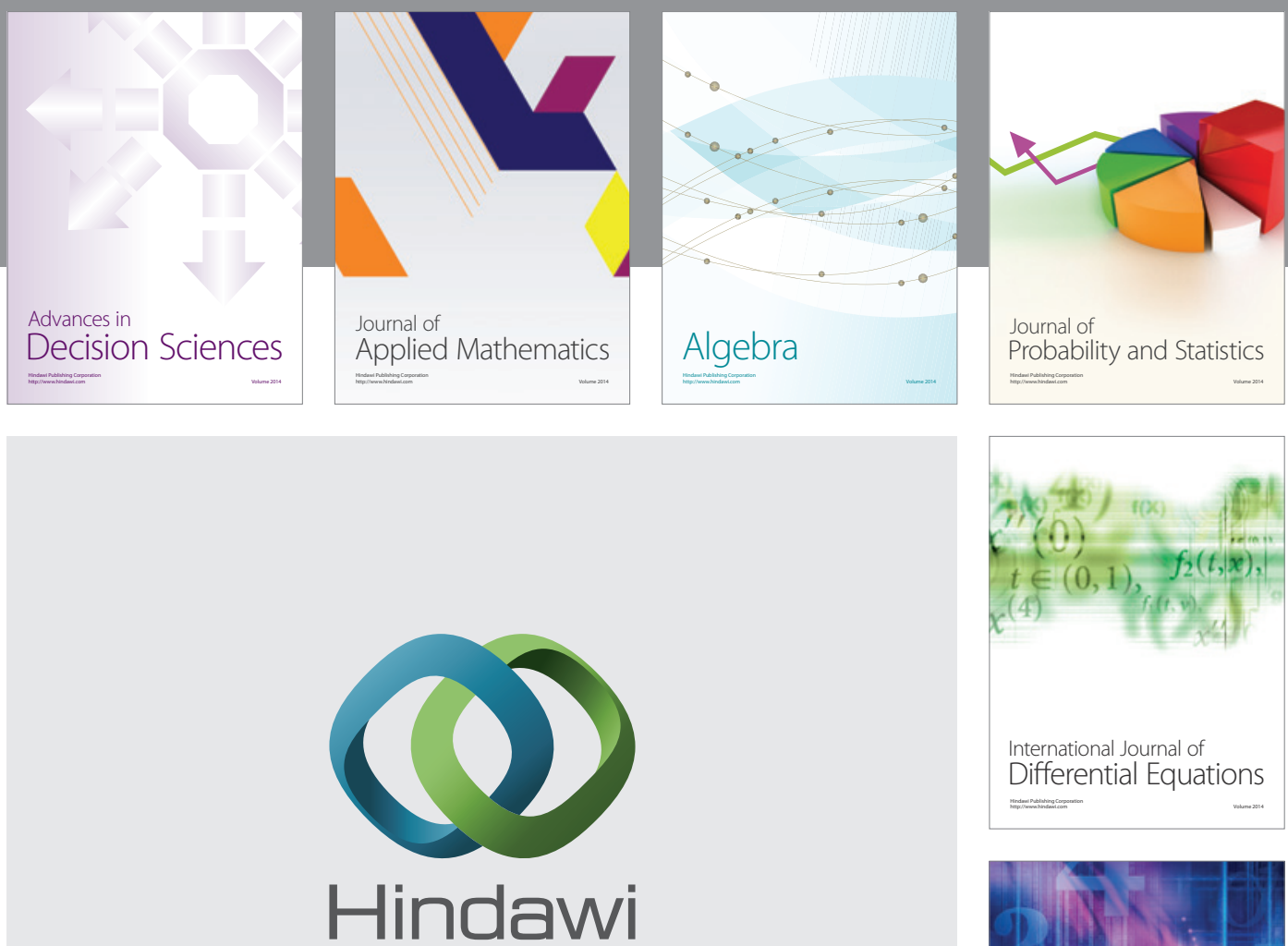

Submit your manuscripts at http://www.hindawi.com
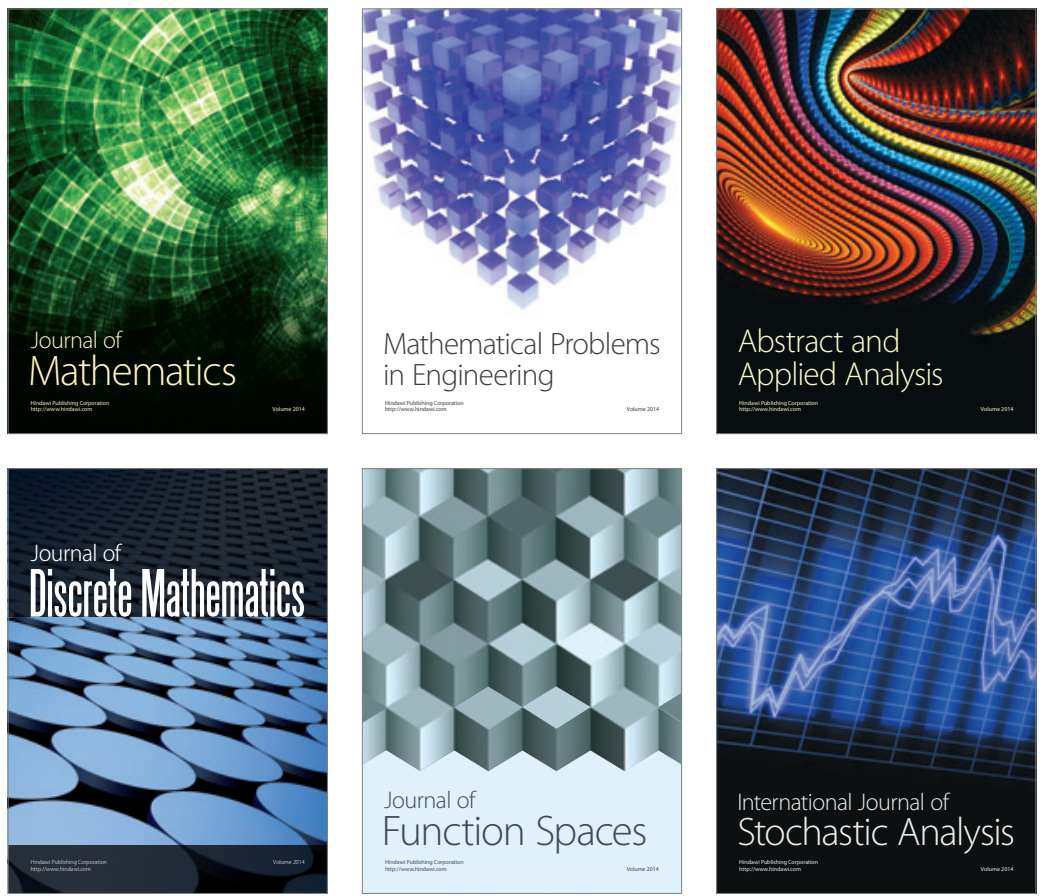

Journal of

Function Spaces

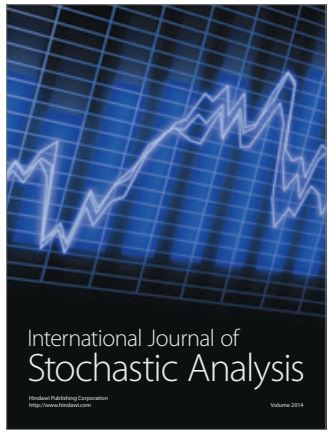

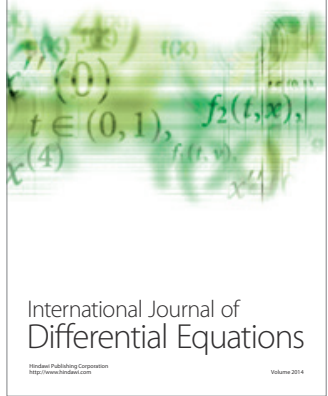
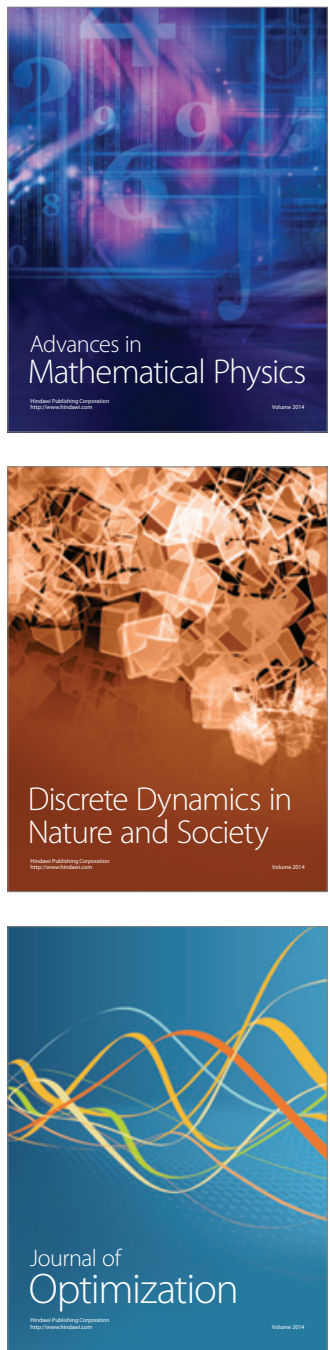